\title{
Theoretical Studies on the Molecular Properties, Toxicity, and Biological Efficacy of 21 New Chemical Entities
}

\author{
Ruby Srivastava*
}

Cite This: ACS Omega 2021, 6, 24891-24901

Read Online

ABSTRACT: New chemical entities (NCEs) such as small molecules and antibody-drug conjugates have strong binding affinity for biological targets, which provide deep insights into structure-specific interactions for the design of future drugs. As structures of drugs increase in complexity, the importance of computational predictions comes into sharp focus. Knowledge of various computational tools enables us to predict the molecular properties, toxicity, and biological efficacy of the drugs and help the medicinal chemists to discover new drugs more efficiently. Newly approved drugs have higher affinities for proteins and nucleic acids and are applied for the treatment of human diseases. We have carried out the computational studies of 21 such NCEs, specifically small molecules and antibody-drug conjugates, and studied the biological efficacy of these drugs. Their bioactivity score and molecular and pharmacokinetic properties were evaluated using online computer software programs, viz., Molinspiration and Osiris

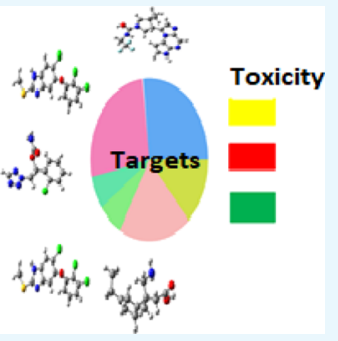
Property Explorer. The SwissTargetPrediction tool was used for the efficient prediction of protein targets for the NCEs. The results indicated higher stability for the drug complexes due to a larger HOMO-LUMO gap. A high electrophilicity index reflects good electrophilic behavior and high reactivity of the drugs. Lipinski's "rule of five" indicated that most of the drug complexes are likely to be orally active. These drugs also showed non-mutagenic, non-tumorigenic, non-irritant, and non-effective reproductive behavior. We hope that these studies will provide an insight into molecular recognition and definitely help the medicinal chemists to design new drugs in future.

\section{INTRODUCTION}

The pharmaceutical industry has been facing significant challenges since the past decade regarding the increased research and development (R\&D) costs, looming patent expirations, and continuously declining number of new drug approvals. ${ }^{1-3}$ Due to the loss of the roaring success of drugs' market share to generic competition, it has become a serious concern that innovation in first-in-class drug discovery has stagnated and the new approved drugs would not be able to replace the losses incurred by expiring patents. Latest data show that the U.S. Food and Drug Administration (FDA) has approved a total of 289 new molecular entities (NMEs), 89 biological license applications (BLAs), and 27 biosimilars between 2010 and 2019, which were evaluated according to curative areas, procedures, administrative routes, high quality selection, approval times, approval numbers by year, and expedited review categories. ${ }^{4}$ Only 19 NMEs were approved in 2007, which has been the lowest number since 1983. The median number of NME and BLA approvals per year was 40, with a low of 21 in 2010 and a high of 59 in 2018. These factors indicate that the pharmaceutical industry should reinvent itself to stop the expected decline in productivity and bring innovative new drugs to the market.

The significant contribution of computational and theoretical studies of quantum chemistry has allowed medicinal chemists to obtain more precise molecular properties and bioactivity of drugs in a shorter time..$^{5-8}$ Due to the evolution of computing data storage and higher processor performance, molecular modeling has been very efficient to solve drug-related issues without compromising the accuracy of the predicted data. ${ }^{9-15}$ Investigation of the mechanism of action of drugs on therapeutic targets can be carried out using structure-activity relationship (SAR) and quantitative structure-activity relationship (QSAR) models. The structures of drugs and their activities or properties are studied using molecular modeling methods and statistical methods. As the structures of small-molecule drugs increase in complexity, computational capability comes into sharp focus. These computational methods are used to design therapeutically important drugs in the pharmaceutical industry. Being divided into two categories as structure-based and ligand-based, the energy evaluation of these two methods depends on force fields or empirical scoring functions. ${ }^{16}$ Therefore, an increased understanding of these ligand-receptor interactions within target molecules is needed to design new drugs with higher specificity.

Fragment-based drug discovery (FBDD) offers the possibility to encode an enormously large number of chemical structures both for similarity-based (2D) and structure-based (3D) de novo

Received: July 14, 2021

Published: September 15, 2021 


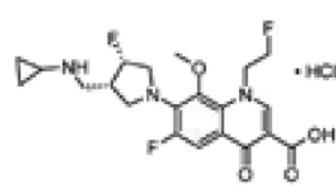

Lascuflaracin hydrochloride.<smiles>[C-]NC(=O)OC(Cn1ncnn1)c1ccccc1Cl</smiles>

Cenobamate

(7)<smiles>CC1C(P)CC(NC(=O)c2cnc3c(c2)CC2(Cc4cccnc4NC2=O)C3)C(=O)N1CC(F)(F)F</smiles>

(12)<smiles>O=[N+]([O-])c1cn2c(n1)OCC(OCc1ccc(OC(F)(F)F)cc1)C2</smiles>

(2)

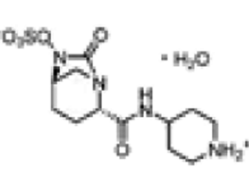

Relebacts

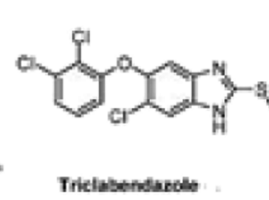

(4)

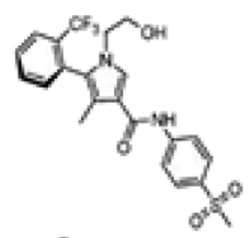

Evexerenene<smiles>CC(C)n1nccc1-c1ncccc1COc1cccc(O)c1C=O</smiles>

(6)

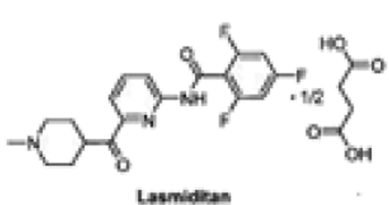

(\$)

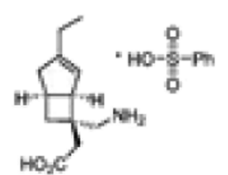

Minogabalin besylate

(9)

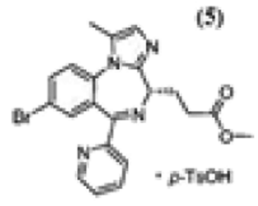

Ponimasolem nesylante

(10)

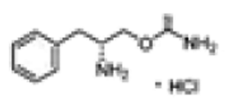

Solinlamfetol

(11)

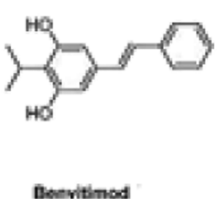

(13)

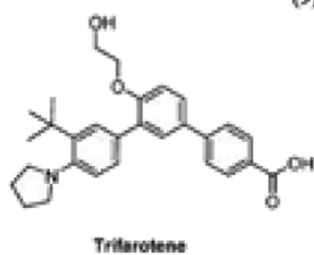

(14)

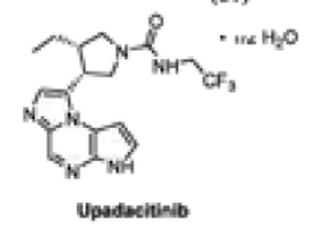

(15)

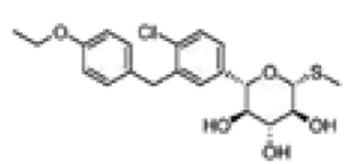

Sotaglifiodin

(16)

(a)

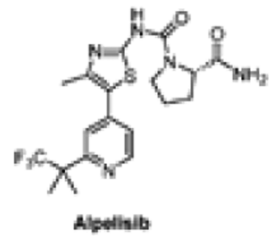

(17)

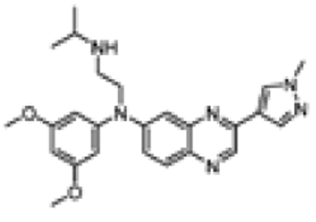

Endufininito

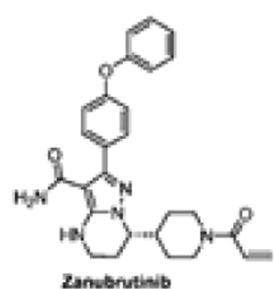

(19)

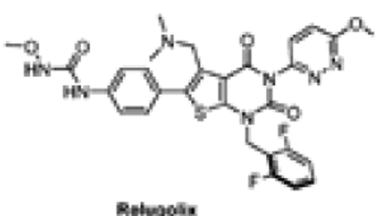

(20)

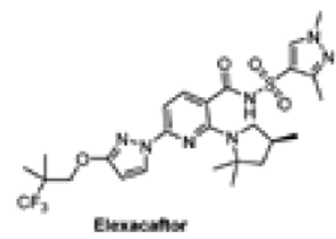

(21)

(b)

Figure 1. Chemical structures of the studied drug complexes (a) (1-16) and (b) 17-21.

design applications. ${ }^{17}$ Various FBDD tools can be used for the search and classification of molecules using physicochemical constraints, ${ }^{18}$ scaffold hopping, ${ }^{19}$ design of molecular architectures based on ligands, ${ }^{20}$ structure-based search within large fragment spaces, $^{21}$ and similarity-driven library design. ${ }^{22}$ Fragment-based drug discovery (FBDD) has also achieved great success in the discovery of highly specific protein kinase inhibitors (PKIs). Protein kinases (PKs) are considered as important drug targets in many diseases due to their vital role in cellular signaling ${ }^{23}$ and in the treatment of multiple diseases. Another novel drug discovery strategy is targeted protein degradation using bifunctional small molecules (PROTACs), which is used to remove specific proteins from within cells. It is a potential strategy which offers therapeutic interventions that have not been achieved with the existing approaches. ${ }^{24}$ Recently, the Pesticide And Drug Fragments (PADFrag) database with a distinct chemo-bioinformatic cross-referencing resource was designed by Yang et al., ${ }^{25}$ which is the first database to define and explore the biologically relevant fragment space by comparing the pesticide and drug fragments. PADFrag combines detailed bioactive fragment data and potential targets and is used to analyze the privileged structures within known bioactive molecules, $a b$ initio molecule library design, core fragment 


\begin{tabular}{|c|c|c|c|c|c|}
\hline a & 8 & a & $80^{8}$ & 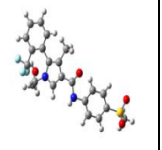 & 3 \\
\hline $\begin{array}{l}\text { Lascufloxacin } \\
\text { (1) }\end{array}$ & $\begin{array}{l}\text { Pretomanid } \\
\text { (2) }\end{array}$ & $\begin{array}{l}\text { Relebactam } \\
\text { (3) }\end{array}$ & $\begin{array}{l}\text { Triclabendazole } \\
\text { (4) }\end{array}$ & $\begin{array}{c}\text { Esaxerenone } \\
(5)\end{array}$ & $\begin{array}{c}\text { Voxelotor } \\
\text { (6) }\end{array}$ \\
\hline & & & & & 急 \\
\hline $\begin{array}{c}\text { Cenobamate } \\
(7)\end{array}$ & $\begin{array}{c}\text { Lasmiditan } \\
(8)\end{array}$ & $\begin{array}{l}\text { Mirogabalin } \\
(9)\end{array}$ & $\begin{array}{c}\text { Remimazolam } \\
(10)\end{array}$ & $\begin{array}{c}\text { Solriamfetol } \\
\text { (11) }\end{array}$ & $\begin{array}{l}\text { Ubrogepant } \\
\text { (12) }\end{array}$ \\
\hline $\log ^{2}$ & 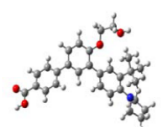 & & & & 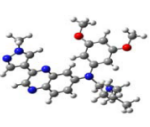 \\
\hline $\begin{array}{c}\text { Benvitimod } \\
\text { (13) }\end{array}$ & $\begin{array}{c}\text { Trifarotene } \\
\text { (14) }\end{array}$ & $\begin{array}{c}\text { Upadacitinib } \\
(15)\end{array}$ & $\begin{array}{c}\text { Sotagliflozin } \\
(16)\end{array}$ & $\begin{array}{c}\text { Alpelisib } \\
(17)\end{array}$ & $\begin{array}{c}\text { Erdafitinib } \\
(18)\end{array}$ \\
\hline 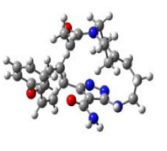 & 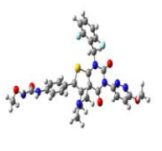 & & & & \\
\hline $\begin{array}{c}\text { Zanubritinib } \\
\text { (19) }\end{array}$ & $\begin{array}{l}\text { Relugolix } \\
\text { (20) }\end{array}$ & $\begin{array}{c}\text { Elexacaftor } \\
(21)\end{array}$ & & & \\
\hline
\end{tabular}

Figure 2. Optimized structures of the studied drug complexes (1-21).

discovery for fragment-based drug design and prediction of fragment interactions, and other drug-related research. ${ }^{25}$

Density functional theory (DFT) has offered a high level of accuracy with lesser computational time. Being cost effective, DFT techniques have provided significant amount of information in a limited time period for biological entities. A drug effect is produced by the molecular recognition of the ligand binding to the active site of a target molecule. It also determines the structure and binding interaction strength of the drug molecule and its target. In a lot of studies, the geometries of drug molecules are predicted efficiently by DFT. DFT also successfully predicts the possible interactions between a drug and a target consisting of ionic and covalent interactions, ${ }^{26,27}$ dipole-dipole interactions, ion-dipole interactions, hydrogen bonding, ${ }^{28,29}$ charge transfer, and hydrophobic interactions. ${ }^{30}$ DFT can also be utilized to explicate the reaction mechanisms of drug and precisely calculate the transition state, which is an important tool for drug design.

Conceptual DFT (CDFT), originally developed by Parr and collaborators, $^{31-37}$ with several global and local reactivity descriptors helps us to understand various physicochemical processes. As global reactivity descriptors are connected with several electronic structure principles, they play a very important role in obtaining the physico-chemical information of the complexes. Various studies ${ }^{38-42}$ have been carried out to predict the chemical properties and the assessment of chemical behavior of drug complexes in order to design and develop new molecules with important biological activities.

The aim of our study is to understand the relationship between the drug structure and its activities and properties responsible for the drug efficiency using CDFT. Out of 11 therapeutic areas such as anti-infective/antibiotic, cardiovascu- lar and hematologic, neurological [central nervous system (CNS)], dermatologic, gastrointestinal, inflammatory and immunologic, metabolic, musculoskeletal, oncologic, reproductive, and respiratory drugs, we have selected 21 drugs from 10 therapeutic areas. Those drugs are lascufloxacin, pretomanid, relebactam, triclabendazole, esaxerenone, voxelotor, cenobamate, lasmiditan, mirogabalin, remimazolam, solriamfetol, ubrogepant, benvitimod, trifarotene, upadacitinib, sotagliflozin, alpelisib, erdafitinib, zanubrutinib, relugolix, and elexacaftor. The chemical structures of these 21 drugs are given in Figure la,b.

\section{RESULTS AND DISCUSSION}

The optimized structures of the 21 selected drugs lascufloxacin (1), pretomanid (2), relebactam (3), triclabendazole (4), esaxerenone (5), voxelotor (6), cenobamate (7), lasmiditan (8), mirogabalin (9), remimazolam (10), solriamfetol (11), ubrogepant (12), benvitimod (13), trifarotene (14), upadacitinib (15), sotagliflozin (16), alpelisib (17), erdafitinib (18), zanubrutinib (19), relugolix (20), and elexacaftor (21) are given in Figure 2, and the SMILES notations and chemical formulas are given in Table 1.

Several chemical reactivity descriptors, such as the chemical potential, global hardness, softness, electrophilicity index, and electronegativity, and HOMO-LUMO (HL) gap, and molecular descriptors have been calculated to understand the physicochemical and pharmacological aspects and the eco-toxicological characteristics of drugs, see Table 2 . The global reactivity descriptors describe the reactivity of the molecule as a whole. The calculated vertical IP values for the drugs are $3.01-8.14 \mathrm{eV}$ and EA values are $0.18-2.27 \mathrm{eV}$ in the water phase. A lower IP of complexes 13,15 , and 19 means a higher probability of losing an 


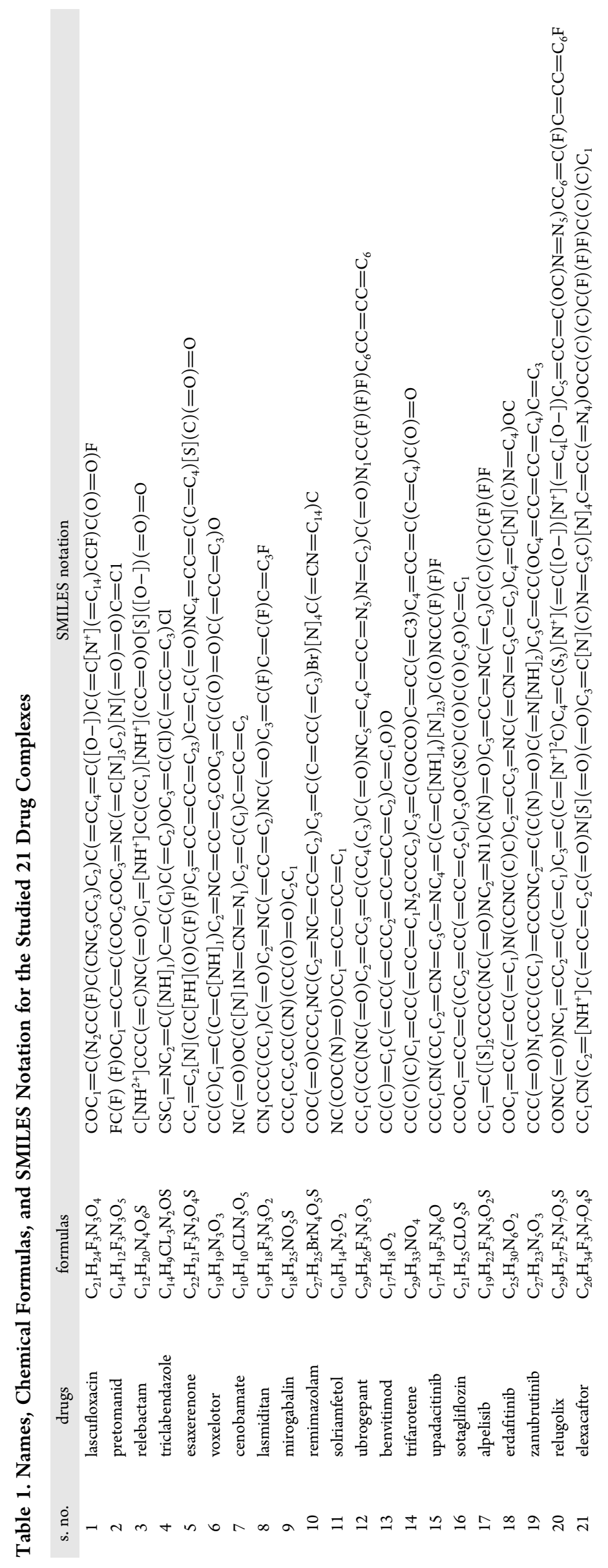


Table 2. Calculated Ionization Energy (IP), Electron Affinity (EA), Electronegativity $(\chi)$, Global Hardness $(\eta)$, Global Softness $(\sigma)$, Global Electrophilicity Index $(\omega)$, and HL Gap in $\mathrm{eV}$ for the Studied 21 Drug Complexes

\begin{tabular}{|c|c|c|c|c|c|c|c|c|}
\hline s. no. & drugs & IP & EA & $\chi$ & $\eta$ & $\Sigma$ & $\omega$ & HL gap \\
\hline 1 & lascufloxacin & 5.78 & 1.57 & 3.67 & 2.10 & 0.2376 & 3.20 & 4.75 \\
\hline 2 & pretomanid & 6.65 & 2.20 & 4.43 & 2.22 & 0.2250 & 4.41 & 4.68 \\
\hline 3 & relebactam & 4.91 & 0.83 & 2.87 & 2.04 & 0.2452 & 2.02 & 2.72 \\
\hline 4 & triclabendazole & 6.02 & 1.18 & 3.60 & 2.42 & 0.2068 & 2.68 & 5.38 \\
\hline 5 & esaxerenone & 6.80 & 1.63 & 4.22 & 2.58 & 0.1935 & 3.44 & 4.93 \\
\hline 6 & voxelotor & 6.20 & 2.25 & 4.23 & 1.97 & 0.2534 & 4.53 & 4.52 \\
\hline 7 & cenobamate & 6.99 & 1.53 & 4.26 & 2.73 & 0.1831 & 3.32 & 6.29 \\
\hline 8 & lasmiditan & 5.90 & 1.86 & 3.88 & 2.02 & 0.2473 & 3.72 & 4.2 \\
\hline 9 & mirogabalin & 6.17 & 0.55 & 3.36 & 2.81 & 0.1780 & 2.01 & 5.61 \\
\hline 10 & remimazolam & 4.35 & 2.27 & 3.31 & 1.04 & 0.4810 & 5.27 & 2.82 \\
\hline 11 & solriamfetol & 6.24 & 0.18 & 3.21 & 3.03 & 0.1649 & 1.70 & 6.85 \\
\hline 12 & ubrogepant & 5.79 & 1.63 & 3.71 & 2.08 & 0.2403 & 3.30 & 5.56 \\
\hline 13 & benvitimod & 4.86 & 1.83 & 3.35 & 1.52 & 0.3299 & 3.69 & 3.68 \\
\hline 14 & trifarotene & 5.72 & 1.70 & 3.71 & 2.01 & 0.2483 & 3.42 & 4.58 \\
\hline 15 & upadacitinib & 3.01 & 1.36 & 2.18 & 0.83 & 0.6050 & 2.89 & 2.22 \\
\hline 16 & sotagliflozin & 5.98 & 0.79 & 3.39 & 2.60 & 0.1926 & 2.21 & 5.63 \\
\hline 17 & alpelisib & 8.14 & 0.08 & 4.11 & 4.03 & 0.1241 & 2.10 & 4.54 \\
\hline 18 & erdafitinib & 5.40 & 2.10 & 3.75 & 1.65 & 0.3031 & 4.26 & 3.85 \\
\hline 19 & zanubrutinib & 4.94 & 1.88 & 3.41 & 1.53 & 0.3272 & 3.80 & 2.21 \\
\hline 20 & relugolix & 5.72 & 1.63 & 3.67 & 2.04 & 0.2446 & 3.30 & 7.19 \\
\hline 21 & elexacaftor & 5.90 & 1.28 & 3.59 & 2.31 & 0.2164 & 2.78 & 6.67 \\
\hline
\end{tabular}

electron. It is clear from the trends observed in IP values that all these drug molecules are capable of electronic charge transfer. A higher EA for complexes 2, 6, 10 and 18 means a higher probability of gaining an electron. Chemical hardness $(\eta)$ is directly correlated to the stability of the molecule, whereas softness $(S)$ is a measure of its reactivity. The calculated chemical hardness $(\eta)$, for each of the chemical species, is found to be lower for all complexes except complex 17. It means a little intramolecular charge transfer for complex 17.

Electronegativity $(\chi)$, which is the negative of the electronic chemical potential $(\mu)$, is a measure of the tendency of a molecule to attract electrons. Electronegativity also measures the attraction of an atom for electrons in a covalent bond. For two unlikely covalently bonded atoms, the shared electrons will be more strongly attracted to the atom of greater electronegativity. In our studied drug complexes, the electronegativity is higher, $2.18-4.43 \mathrm{eV}$. The chemical potential $(\mu)$, which is a measure of the tendency of an electron to escape, is found to follow the opposite trend for the studied complexes. The electrophilicity index is considered as a better descriptor to provide information on electron transfer (chemical potential) and stability (hardness). Also, it has both the ability of an electrophile to acquire an additional electronic charge and the resistance of the system to exchange an electronic charge with the environment. A higher electrophilicity index means a highly reactive nature of the drug molecules. It also measures the propensity of chemical species to accept electrons. A high value of electrophilicity index describes a good electrophilic behavior, while a small value of electrophilicity index describes a good nucleophilic behavior. The electrophilicity index is higher for most of the complexes, $1.70-5.27 \mathrm{eV}$.

The reactivity of the molecule can be demonstrated by the distribution of the orbital frontiers which is predicted with the help of the frontier molecular orbital (FMO) theory. The frontier orbital gap helps to characterize the chemical reactivity and kinetic stability ${ }^{43}$ of the molecules. The HOMO and the LUMO determine the way by which it interacts with other species. The HOMO energy indicates the tendency of a molecule to donate electrons to empty molecular orbitals with a low energy of convenient molecules and the LUMO energy indicates the ability to accept electrons. The higher HL gap of the studied drug complexes reflects the high chemical stability and the low reactivity of the complexes.

According to Lipinski's "rule of five", a candidate molecule will likely be orally active, if (i) the molecular weight (MW) < 500, (ii) the calculated octanol/water partition coefficient (log $P)<5$, (iii) there were fewer than 5 hydrogen bond donors (HBD) ( $\mathrm{OH}$ and $\mathrm{NH}$ groups), and (iv) there are less than 10 hydrogen bond acceptors (HBA) (notably $\mathrm{N}$ and $\mathrm{O}$ ). ${ }^{44}$ Molecular properties such as $\log P$, topological polar surface area (TPSA), HBD and HBA, and MW, and violations of Lipinski's rule of five were determined to evaluate the druglikeness and drug score of the studied drugs. The Ghose, Pritchett, and Crippen method ${ }^{45}$ was used to calculate the $\log P$ values. $\log P$ is related to the lipophilicity of compounds and is useful for prediction of the absorption of drugs across the intestinal epithelium. In our studied drug complexes, only complexes 12 and 20 have $\mathrm{MW}>500$. The violation of $\log \mathrm{P}$ values is seen in complexes 1 and 14 (a negative value for $\log P$ indicates higher affinity for the aqueous phase), while other drug complexes show $\log P(0.39-4.5)<5$, which is the upper limit for drug penetration through biomembranes. Thus, all these compounds have good bioavailability.

Furthermore, HBA (as total number of nitrogen and oxygen atoms) is less than 10 for all the complexes except complexes 3 and 21 , and HBD (as total number of nitrogen-hydrogen and oxygen-hydrogen bonds) is less than 5 for all the drugs except complex 3. The TPSA is calculated from the surfaces which are occupied by the oxygen and nitrogen atoms and by the hydrogen atoms attached to them. Thus, TPSA is closely linked to the hydrogen bonding potential of a compound. ${ }^{46}$ Molecules with TPSA values > $140 \AA^{2}$ or more would show poor intestinal absorption, ${ }^{47}$ and TPSA $<90 \AA^{2}$ is needed to penetrate the blood-brain barrier (and thus act on receptors in the CNS). 
Table 3. CLP- $\operatorname{cog} P, S$-Solubility, MW-Molecular Weight, TPSA-Topological Polar Surface Area, HBA-Number of Hydrogen Bond Acceptors, and HBD-Number of Hydrogen Bond Donors of the Studied 21 Drug Complexes

\begin{tabular}{|c|c|c|c|c|c|c|c|}
\hline & & CLP & $S$ & MW & TPSA & HBA & $\mathrm{HBD}$ \\
\hline s. no. & drugs & $\leq 5$ & & $<500$ & & $<10$ & $<5$ \\
\hline 1 & lascufloxacin & -5.55 & -3.43 & 439 & 88.74 & 7 & 2 \\
\hline 2 & pretomanid & 2.64 & -3.56 & 361 & 105.2 & 8 & 0 \\
\hline 3 & relebactam & 4.15 & -0.13 & 398 & 136.0 & 10 & 5 \\
\hline 4 & triclabendazole & 5.19 & -6.95 & 358 & 63.21 & 3 & 1 \\
\hline 5 & esaxerenone & 2.94 & -4.63 & 466 & 96.78 & 6 & 2 \\
\hline 6 & voxelotor & 2.86 & -3.58 & 352 & 95.44 & 6 & 3 \\
\hline 7 & cenobamate & 0.93 & -2.07 & 267 & 95.92 & 7 & 2 \\
\hline 8 & lasmiditan & 2.67 & -4.45 & 377 & 62.3 & 5 & 1 \\
\hline 9 & mirogabalin & -0.59 & -2.37 & 211 & 63.32 & 3 & 3 \\
\hline 10 & remimazolam & 3.11 & -5.02 & 440 & 69.04 & 6 & 1 \\
\hline 11 & solriamfetol & 0.39 & -2.23 & 194 & 78.34 & 4 & 4 \\
\hline 12 & ubrogepant & 2.43 & -5.43 & 551 & 104.2 & 8 & 2 \\
\hline 13 & benvitimod & 3.81 & -3.15 & 254 & 40.45 & 2 & 2 \\
\hline 14 & trifarotene & 5.95 & -7.49 & 459 & 70.00 & 5 & 2 \\
\hline 15 & upadacitinib & 1.25 & -4.4 & 382 & 81.49 & 7 & 3 \\
\hline 16 & sotagliflozin & 3.6 & -4.44 & 424 & 104.4 & 5 & 3 \\
\hline 17 & alpelisib & 3.81 & -4.95 & 442 & 101.21 & 7 & 3 \\
\hline 18 & erdafitinib & 1.52 & -4.52 & 446 & 77.33 & 8 & 3 \\
\hline 19 & zanubrutinib & 3.39 & -4.32 & 475 & 113.3 & 8 & 1 \\
\hline 20 & relugolix & 3.7 & -8.21 & 623 & 157.4 & 8 & 4 \\
\hline 21 & elexacaftor & 4.5 & -3.9 & 597 & 132.6 & 12 & 2 \\
\hline
\end{tabular}

Table 4. Bioactivity Score of the Compounds according to Molinspiration Cheminformatics Software; GPCR, Ligand Ion Channel Modulator (ICM), Kinase Inhibitor (KI), Nuclear Receptor Ligand (NCL), Protease Inhibitor (PI), and Enzyme Inhibitor (EI) for the Studied 21 Drug Complexes

\begin{tabular}{|c|c|c|c|c|c|c|c|}
\hline \multirow[b]{2}{*}{ s. no. } & \multirow[b]{2}{*}{ complexes } & \multicolumn{6}{|c|}{ drug-likeness } \\
\hline & & GPCR & ICM & $\mathrm{KI}$ & NCL & PI & EI \\
\hline 1 & lascufloxacin & 0.33 & 0.23 & 0.01 & -0.53 & 0.15 & 0.39 \\
\hline 2 & pretomanid & 0.10 & 0.02 & -0.24 & -0.17 & -0.19 & 0.20 \\
\hline 3 & relebactam & -0.06 & -0.07 & -0.28 & -0.49 & 0.06 & -0.03 \\
\hline 4 & triclabendazole & -0.14 & -0.31 & 0.01 & -0.39 & -0.56 & -0.05 \\
\hline 5 & esaxerenone & -0.06 & -0.21 & -0.11 & -0.01 & -0.08 & 0.07 \\
\hline 6 & voxelotor & 0.12 & 0.11 & 0.19 & 0.31 & -0.09 & 0.41 \\
\hline 7 & cenobamate & 0.34 & -0.18 & -0.07 & -0.4 & 0.12 & 0.37 \\
\hline 8 & lasmiditan & 0.29 & -0.17 & 0.02 & -0.31 & 0.21 & 0.09 \\
\hline 9 & mirogabalin & -0.04 & 0.92 & -0.62 & -0.24 & -0.14 & 0.33 \\
\hline 10 & remimazolam & 0.20 & -0.14 & -0.5 & -0.63 & -0.26 & -0.05 \\
\hline 11 & solriamfetol & 0.20 & 0.53 & 0.09 & -0.23 & 0.38 & 0.20 \\
\hline 12 & ubrogepant & 0.52 & -0.22 & -0.07 & -0.14 & 0.15 & 0.18 \\
\hline 13 & benvitimod & -0.38 & -0.18 & -0.34 & -0.34 & -0.56 & -0.14 \\
\hline 14 & trifarotene & 0.14 & 0.09 & 0.11 & 0.41 & 0.01 & 0.14 \\
\hline 15 & upadacitinib & 0.7 & 0.23 & 0.68 & -0.14 & 0.22 & 0.33 \\
\hline 16 & sotagliflozin & 0.07 & -0.19 & -0.33 & 0.02 & 0.1 & 0.26 \\
\hline 17 & alpelisib & 0.37 & -0.15 & 0.32 & -0.09 & 0.18 & 0.24 \\
\hline 18 & erdafitinib & 0.2 & 0.02 & 0.34 & -0.33 & -0.06 & 0.05 \\
\hline 19 & zanubrutinib & 0.18 & -0.19 & 0.03 & -0.25 & 0.02 & 0.06 \\
\hline 20 & relugolix & -0.25 & -0.4 & -0.28 & -1.17 & -0.22 & 0.05 \\
\hline 21 & elexacaftor & -0.12 & -0.73 & -0.42 & -0.48 & -0.14 & -0.20 \\
\hline
\end{tabular}

The studied drug complexes $1,4,8,9,10,11,13,14,15$, and 18 have TPSA < 90, while other drug complexes have $90<$ TPSA > 140 , except complex $20\left(157.4 \AA^{2}\right)$. It should be noted that the $\log P$ and TPSA values are not sufficient to predict the oral absorption of a drug. ${ }^{48}$ The drug complexes need to respect the rule of five. $\log P$ can be correlated with PSA when the potential drugs are evaluated according to Hughes et al. ${ }^{49}$ who proposed the criteria as $\log P<4$ and PSA $>75 \AA^{2}$. As is known, two or more violations of the rule of five suggest the likelihood of bioavailability problems, ${ }^{49}$ see Table 3 . According to the Lipinski rule, most of the drug complexes showed good bioavailability.

Properties such as hydrogen bonding, hydrophobicity, electronic distribution, flexibility, size of molecules, and the presence of various characteristics of pharmacophores have an effect on the behavior of the molecule in a biological medium, 


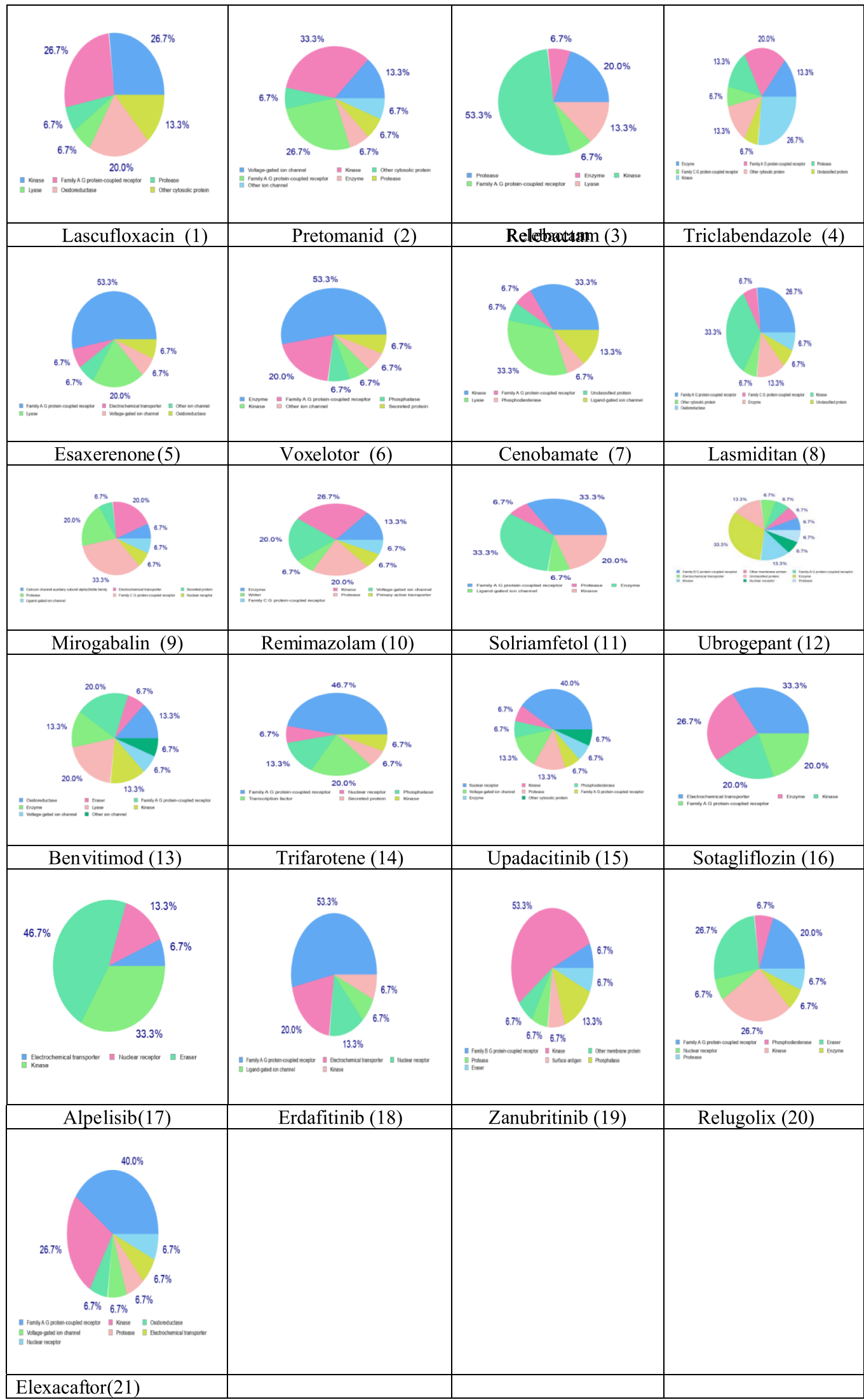

Figure 3. Predicted biological targets of the studied drug complexes (1-21) using the SwissTargetPrediction online tool.

including the bioavailability, reactivity, toxicity, protein affinity, metabolic stability, transport properties, and many more. The activity of all standard compounds and drugs has been rigorously analyzed according to the following four criteria of successful drug activity: activity on the $G$ protein-coupled receptor (GPCR) ligand, modulation of ion channels, inhibition of 
Table 5. DL-Drug-Likeliness, DS—Drug Score, MUT-Mutagenicity, TUMO-Tumorigenicity, IRRI-Irritant Effect, and REP-Reproductive Effect for the Studied 21 Drug Complexes

\begin{tabular}{|r|l|l|l|l|l|l|l|}
\hline S.No & Drugs & DL & DS & MUT & TUMO & IRRI & REP \\
\hline & & & & & & & \\
\hline 1 & Lascufloxacin & 1.92 & 0.72 & & & & \\
\hline 2 & Pretomanid & -13.2 & 0.40 & & & & \\
\hline 3 & Relebactan & 5.2 & 0.33 & & & & \\
\hline 4 & Triclabendazole & 2.17 & 0.10 & & & & \\
\hline 5 & Esaxerenon & -3.35 & 0.31 & & & & \\
\hline 6 & Voxilotor & -0.8 & 0.52 & & & & \\
\hline 7 & Cenobamate & -0.8 & 0.52 & & & & \\
\hline 8 & Lasmiditan & 6.01 & 0.71 & & & & \\
\hline 9 & Mirogabelin & 0.05 & 0.72 & & & & \\
\hline 10 & Renimazolam & -6.73 & 0.29 & & & & \\
\hline 11 & Solriamfetol & 1.68 & 0.88 & & & & \\
\hline 12 & Ubrogepant & -0.87 & 0.29 & & & & \\
\hline 13 & Benritimod & -0.51 & 0.55 & & & & \\
\hline 14 & Trifarotene & -5.09 & 0.05 & & & & \\
\hline 15 & Upadacinitib & -1.97 & 0.41 & & & & \\
\hline 16 & Sotagliflozin & 0.2 & 0.49 & & & & \\
\hline 17 & Alpilisib & -4.79 & 0.31 & & & & \\
\hline 18 & Erdafinitib & 2.5 & 0.28 & & & & \\
\hline 19 & Zenubritinib & 5.22 & 0.60 & & & & \\
\hline 20 & Relugolix & 5.3 & 0.22 & & & & \\
\hline 21 & Elexacafor & -4.0 & 0.22 & & & & \\
\hline & & & & & & \\
\hline
\end{tabular}

kinases, proteases, and other enzymes, and activity on nuclear receptor ligands. Molinspiration Cheminformatics software was used to calculate the drug activities of the 21 drug complexes and they are reported in Table 4. A molecule having a bioactivity score more than 0.00 is most likely to exhibit considerable biological activities, while molecules with values -0.50 to 0.00 are expected to be moderately active, and if the score is less than -0.50 it is presumed to be inactive. The results clearly reveal that the physiological actions of drug complexes might involve multiple mechanisms and could be due to the interactions with GPCR ligands, nuclear receptor ligands, and inhibitors of proteases and other enzymes. These bioactivity scores are highly suggestive of a moderate interaction with all drug targets.

To further predict the higher efficiency and probable prediction of protein targets for the studied NCEs, we used the SwissTargetPrediction online tool. We found that the complexes $2,3,4,8,10,17,19$, and 20 have higher probability for kinase inhibitors, complexes 5, 9, 11, 14, and 18 for GPCR, and complexes 6 and 21 for enzyme inhibitors as potential targets. Complexes 15 and 16 have higher probability for nuclear receptors and electrochemical transporters as potential targets. Complexes 1 and 21 have estimated equal probability for kinase and GPCR as targets, while complex 13 has the estimated equal probability for lyase and GPCR as targets, see Figure 3.

Nowadays, structure-based designs are very common, but because of inappropriate absorption, distribution, metabolism, elimination, and toxicity (ADMET) properties, many potential drugs do not reach the clinic. Therefore, the risks of toxicity (mutagenicity, tumorigenicity, irritation, reproductive effect) and the physicochemical properties (drug-likeness and drug score) of the studied 21 drug compounds were calculated using Osiris software, see Table 5.

The toxicity risk predictor locates fragments in a molecule, indicating a potential toxicity risk. These alerts indicate that the structure drawn can be harmful concerning the specified risk category. The color code for these categories are red, green, and yellow. ${ }^{50}$ Green color suggests a low toxic potential, yellow means mild toxicity, and red color indicates a high probability for toxicity. The results clearly indicate that all compounds except complexes $3,4,14$, and 18 would be safe and expected to show low or no toxicity regarding mutagenicity, tumorigenicity, irritation, and effect on the reproductive system. According to the data presented in Table 5, following are the toxic effects of the above-mentioned drug complexes: 3 (highly toxicmutagenic and highly toxic-reproductive), 4 (slightly toxicmutagenic, highly toxic-irritant, and highly toxic-reproductive), 14 (highly toxic-mutagenic and highly toxic-tumorigenic), and 18 (mild-mutagenic). At the same time, most of the drugs have shown good results in screening with best drug score values ( $\mathrm{DS}=0.62-0.83$ ), compared to the other drugs having lower values in Table 5 .

\section{CONCLUSIONS}

The results indicate higher chemical stability for the studied 21 drug complexes. Good electrophilic behavior has been observed for the studied drug complexes due to the higher electrophilicity index. Most of the studied drugs are non-mutagenic, nontumorigenic, non-irritant, and without effects on reproduction, and they have good drug score values. The results indicate that the physiological actions of drug complexes might involve multiple mechanisms and could be due to the interactions with kinase inhibitors, GPCR ligands, nuclear receptor ligands, and inhibitors of proteases and other enzymes. The higher HL gap of the studied drug complexes predicted a high chemical stability and a low reactivity of the complexes.

In the past few years, QSAR-based computational models have been used to predict the physicochemical properties, druglikeliness, and toxicity of drugs. On the basis of these data, recently developed artificial intelligence (AI) approaches, such as deep learning and relevant modeling approaches, are implemented for the safety and efficacy evaluations of drug molecules. The continued improvement of machine learning (ML) methods which compete with standard approaches and expert and skill has changed the world of computational medicinal chemistry. In near future, the basic scientific research 
will move to a place where knowledge and understanding will overtake the quantitative results.

\section{COMPUTATIONAL DETAILS}

The calculations have been performed with two different basis sets, $\mathrm{M} 06^{51} / 6-31 \mathrm{G}^{* *}$ and $\mathrm{B} 3 \mathrm{LYP}^{52} / 6-31 \mathrm{G}^{* *}$ from $\mathrm{G}^{*} 6^{53}$ software suites. Vibrational frequency analysis has been carried out for all the optimized structures and there is no negative frequency for the lowest energy structures. Finally, the lowest energy minima with $\mathrm{M06} / 6-31 \mathrm{G}^{* *}$ basis set values were selected for further calculations. The energy values from the two methods are reported in Table S1. The calculations were performed using water as a solvent by the integral equation formalism-polarized continuum model (IEF-PCM) according to the SMD solvation model. ${ }^{54}$ GaussView $^{55}$ was used to draw the optimized structures of drug molecules. The output (pdb) structures were used as input to generate the SMILES notation from the Online SMILES Translator and Structure File Generator tool.

Chemical reactivity theory was been applied to calculate the global descriptors for these NCEs. Molinspiration ${ }^{56}$ software (www.molinspiration.com) was used to calculate various molecular properties and to predict the bioactivity score for drug targets including enzymes and nuclear receptors, kinase inhibitors, GPCR ligands, and ion channel modulators. Molecular properties such as log P, TPSA, HBD and HBA, and MW weight were also calculated. Lipinski's rule ${ }^{44}$ of five was applied to evaluate the drug-likeness, and the bioactivity score of the drugs were also compared. Using Molinspiration Cheminformatics, all pharmacokinetic parameters were evaluated to predict the bioactivity of compounds. The online SwissTargetPrediction ${ }^{57}$ tool was used for the efficient prediction of protein targets for the NCEs.

Osiris Property Explorer ${ }^{58}$ was used to determine pharmacokinetic parameters such as toxicity potential, solubility, and overall drug-likeness of these NCEs. The results of virtual screening were evaluated and color-coded either green or red for properties such as the mutagenic effect, irritant effect, tumorigenicity, and effect on the reproductive system. Red color indicates the high risk of undesired effects, while green color shows drug-conformity behavior, compatibility, and safety in vivo.

\section{DATA AVAILABILITY}

The optimized structure (CIF) coordinates of all 21 drug complexes are given in the Supporting Information.

\section{ASSOCIATED CONTENT}

\section{SI Supporting Information}

The Supporting Information is available free of charge at https://pubs.acs.org/doi/10.1021/acsomega.1c03736.

Formulas used, optimized energies (au) of the studied 21 drug complexes by M06/6-31G** and B3LYP/6-31G** basis sets, and optimized structure (CIF) coordinates of the studied 21 drug complexes (PDF)

\section{AUTHOR INFORMATION}

\section{Corresponding Author}

Ruby Srivastava - Bioinformatics, CSIR-CCMB, Hyderabad 500007, India; 이이.org/0000-0002-2367-0176;

Email: amitruby1@gmail.com, ruby@ccmb.res.in
Complete contact information is available at:

https://pubs.acs.org/10.1021/acsomega.1c03736

\section{Author Contributions}

The manuscript is solely written by the corresponding author.

Notes

The author declares no competing financial interest.

\section{ACKNOWLEDGMENTS}

R.S. acknowledges the financial assistance by the DST WOSA project (SR/WOS-A/CS-69/2018). R.S. is thankful to her mentor Dr. Shrish Tiwari, Bioinformatics, CSIR-Centre for Cellular and Molecular Biology, and Dr. G. Narahari Sastry, Director, CSIR-NEIST for the technical support.

\section{REFERENCES}

(1) Ruffolo, R. R. Why Has R\&D Productivity Declined in the Pharmaceutical Industry? Expet Opin. Drug Discov. 2006, 1, 99-102.

(2) Scannell, J. W.; Blanckley, A.; Boldon, H.; Warrington, B. Diagnosing the Decline in Pharmaceutical R\&D Efficiency. Nat. Rev. Drug Discovery 2012, 11, 191-200.

(3) Paul, S. M.; Mytelka, D. S.; Dunwiddie, C. T.; Persinger, C. C.; Munos, B. H.; Lindborg, S. R.; Schacht, A. L. How to Improve R\&D Productivity: The Pharmaceutical Industry's Grand Challenge. Nat. Rev. Drug Discovery 2010, 9, 203-214.

(4) Brown, D. G.; Wobst, H. J. J. Med. Chem. 2021, 64, 2312-2338.

(5) Lin, X.; Li, X.; Lin, X. A Review on Applications of Computational Methods in Drug Screening and Design. Molecules 2020, 25, 13751392.

(6) Sliwoski, G.; Kothiwale, S.; Meiler, J.; Lowe, E. W., Jr. Computational Methods in Drug Discovery. Pharmacol. Rev. 2014, $66,334-395$.

(7) Schaduangrat, N.; Lampa, S.; Simeon, S.; Gleeson, M. P.; Spjuth, O.; Nantasenamat, C. Towards reproducible computational drug discovery. J. Cheminf. 2020, 12, 9-39.

(8) Willems, H.; De Cesco, S.; Svensson, F. Computational Chemistry on a Budget: Supporting Drug Discovery with Limited Resources. J. Med. Chem. 2020, 63, 10158-10169.

(9) Medina-Franco, J. L.; Sánchez-Cruz, N.; López-López, E.; DíazEufracio, B. I. Progress on open chemoinformatic tools for expanding and exploring the chemical space. J. Comput. Aided Mol. Des. 2021, 18, $1-14$.

(10) Patel, H.; Kukol, A. Integrating molecular modelling methods to advance influenza A virus drug discovery. Drug Discov. Today 2021, 26, 503-510.

(11) Shen, C.; Weng, G.; Zhang, X.; Leung, L.-H.; Yao, X.; Pang, J.; Chai, X.; Li, D.; Wang, E.; Cao, D.; Hou, T. Accuracy or novelty: what can we gain from target-specific machine-learning-based scoring functions in virtual screening? Briefings Bioinf. 2021, 63, bbaa410.

(12) Medina-Franco, J. L.; Martinez-Mayorga, K.; Fernández-de Gortari, E.; Kirchmair, J.; Bajorath, J. Rationality over fashion and hype in drug design. F1000Research 2021, 10, 397.

(13) Holderbach, S.; Adam, L.; Jayaram, B.; Wade, R. C.; Mukherjee, G. RASPD+: Fast Protein-Ligand Binding Free Energy Prediction Using Simplified Physicochemical Features. Front. Mol. Biosci. 2020, 7, 601065-601079.

(14) Singh, N.; Decroly, E.; Khatib, A.-M.; Villoutreix, B. O. Structurebased drug repositioning over the human TMPRSS2 protease domain: search for chemical probes able to repress SARS-CoV-2 Spike protein cleavages. Eur. J. Pharm. Sci. 2020, 153, 105495-105505.

(15) Vanpoucke, D. E. P.; van Knippenberg, O. S. J.; Hermans, K.; Bernaerts, K. V.; Mehrkanoon, S. Small data materials design with machine learning: When the average model knows best. J. Appl. Phys. 2020, 128, 054901.

(16) Smith, J. S.; Roitberg, A. E.; Isayev, O. Transforming Computational Drug Discovery with Machine Learning and AI. ACS Med. Chem. Lett. 2018, 9, 1065-1069. 
(17) Wang, Z.-Z.; Shi, X.-X.; Huang, G.-Y.; Hao, G.-F.; Yang, G.-F. Fragment-based drug design facilitates selective kinase inhibitor discovery. Trends Pharmacol. Sci. 2021, 42, 551-565.

(18) Pärn, J.; Degen, J.; Rarey, M. Exploring fragment spaces under multiple physicochemical constraints. J. Comput. Aided Mol. Des. 2007, 21, 327-340.

(19) Maass, P.; Schulz-Gasch, T.; Stahl, M.; Rarey, M. Recore: A fast and versatile method for scaffold hopping based on small molecule crystal structure conformations. J. Chem. Inf. Model. 2007, 47, 390-399.

(20) Schneider, G.; Lee, M.-L.; Stahl, M.; Schneider, P. De novo design of molecular architectures by evolutionary assembly of drugderived building blocks. J. Comput. Aided Mol. Des. 2000, 14, 487-494.

(21) Degen, J.; Rarey, M. FlexNovo: Structure-based searching in large fragment spaces. ChemMedChem 2006, 1, 854-868.

(22) Fischer, J. R.; Lessel, U.; Rarey, M. LoFT: Similarity-Driven Multiobjective Focused Library Design. J. Chem. Inf. Model. 2010, 50, $1-21$.

(23) Manning, G.; Whyte, D. B.; Martinez, R.; Hunter, T.; Sudarsanam, $\mathrm{S}$. The protein kinase complement of the human genome. Science 2002, 298, 1912-1934.

(24) Churcher, I. Protac-Induced Protein Degradation in Drug Discovery: Breaking the Rules or Just Making New Ones? J. Med. Chem. 2018, 61, 444-452.

(25) Yang, J.-F.; Wang, F.; Jiang, W.; Zhou, G.-Y.; Li, C.-Z.; Zhu, X.-L.; Hao, G.-F.; Yang, G.-F. PADFrag: A Database Built for the Exploration of Bioactive Fragment Space for Drug Discovery. J. Chem. Inf. Model. 2018, 58, 1725-1730.

(26) Jemmis, E. D.; Giju, K. T.; Leszczynski, J. Ionic to covalent bonding: a density functional theory study of linear and bent X2Y3 monomers (X=B, Al, Ga, In; Y=O, S, Se). Electron. J. Theor. Chem. 1997, $2,130-138$.

(27) Mó, O.; Yáñez, M.; Eckert-Maksíc, M.; Maksić, Z. B.; Alkorta, I.; Elguero, J. Periodic trends in bond dissociation energies. A theoretical study. J. Phys. Chem. A 2005, 109, 4359-4365.

(28) Allen, E. C.; Beers, K. J. Ab initio study of the binding strength of POSS-cation complexes. Polymer 2005, 46, 569-573.

(29) Tuma, C.; Boese, A. D.; Handy, N. C. Predicting the binding energies of H-bonded complexes: A comparative DFT study. Phys. Chem. Chem. Phys. 1999, 1, 3939-3947.

(30) Reimers, J. R.; CAI, Z.-L.; Bilić, A.; Hush, N. S. The appropriateness of density-functional theory for the calculation of molecular electronics properties. Ann. N. Y. Acad. Sci. 2003, 1006, 235251.

(31) Parr, R. G.; Yang, W. Density Functional Theory of Atoms and Molecules; Oxford University Press: Oxford, 1989; pp 1-333.

(32) Kohn, W.; Becke, A. D.; Parr, R. G. Density Functional Theory of Electronic Structure. J. Phys. Chem. 1996, 100, 12974-12980.

(33) Ayers, P. W.; Yang, W. Density Functional Theory. In Computational Medicinal Chemistry for Drug Discovery; Bultinck, P., de Winter, H., Langenaeker, W., Tollenaere, J. P., Eds.; Dekker: New York, 2003; pp 1-571.

(34) Parr, R. G.; Yang, W. Density-functional theory of the electronic structure of molecules. Annu. Rev. Phys. Chem. 1995, 46, 701-728.

(35) Sarkar, U.; Chattaraj, P. K. Reactivity Dynamics. J. Phys. Chem. A 2021, 125, 2051-2060.

(36) Chattaraj, P. K.; Poddar, A.; Maiti, B. Chemical Reactivity and Dynamics within a Density-based Quantum Mechanical Framework. In Reviews In Modern Quantum Chemistry: A Celebration of the Contributions of Robert Parr; Sen, K. D., Ed.; World Scientific: Singapore, 2002; pp 1-871.

(37) Chattaraj, P. K.; Nath, S.; Maiti, B. Reactivity Descriptors. Computational Medicinal Chemistry for Drug Discovery; Taylor \& Francis: New York, 2003; pp 1-295.

(38) Roy, D. R.; Sarkar, U.; Chattaraj, P. K.; Mitra, A.; Padmanabhan, J.; Parthasarathi, R.; Subramanian, V.; Van Damme, S.; Bultinck, P. Analyzing Toxicity Through Electrophilicity. Mol. Diversity 2006, 10, 119-131.
(39) Roy, D. R.; Pal, N.; Mitra, A.; Bultinck, P.; Parthasarathi, R.; Subramanian, V.; Chattaraj, P. K. An atom counting strategy towards analyzing the biological activity of sex hormones. Eur. J. Med. Chem. 2007, 42, 1365-1369.

(40) Bououden, W.; Benguerba, Y. Computational Quantum Chemical Study, Drug-Likeness and In Silico Cytotoxicity Evaluation of Some Steroidal Anti-Inflammatory Drugs. J. Drug Deliv. Therapeut. 2020, 10, 68-74.

(41) Reenu; Vikas. Electron-correlation based externally predictive QSARs for mutagenicity of nitrated-PAHs in Salmonella typhimurium TA100. Ecotoxicol. Environ. Saf. 2014, 101, 42-50.

(42) Srivastava, R. Chemical reactivity theory (CRT) study of small drug-like biologically active molecules. J. Biomol. Struct. Dyn. 2021, 39, 943-952.

(43) Elsharkawy, E. R.; Almalki, F.; Ben Hadda, T.; Rastija, V.; Lafridi, H.; Zgou, H. DFT calculations and POM analyses of cytotoxicity of some flavonoids from aerial parts of Cupressus sempervirens: Docking and identification of pharmacophore sites. Bioorg. Chem. 2020, 100, 103850-103879.

(44) Lipinski, C. A.; Lombardo, F.; Dominy, B. W.; Feeney, P. J. Experimental and computational approaches to estimate solubility and permeability in drug discovery and development settings. Adv. Drug Deliv. Rev. 1997, 23, 3-25.

(45) Ghose, A. K.; Pritchett, A.; Crippen, G. M. Atomic physicochemical parameters for three-dimensional structure directed quantitative structure-activity relationships. III: Modeling hydrophobic interactions. J. Comput. Chem. 1988, 9, 80-90.

(46) Deconinck, E.; Ates, H.; Callebaut, N.; Van Gyseghem, E.; Vander Heyden, Y. Evaluation of chromatographic descriptors for the prediction of gastro-intestinal absorption of drugs. J. Chromatogr. A 2007, 1138, 190-202.

(47) Daina, A.; Michielin, O.; Zoete, V. SwissADME: a free web tool to evaluate pharmacokinetics, drug-likeness and medicinal chemistry friendliness of small molecules. Sci. Rep. 2017, 7, 42717-42730.

(48) Edmondson, S. D.; Yang, B.; Fallan, C. Proteolysis targeting chimeras (PROTACs) in "beyond rule-of-five" chemical space: Recent progress and future challenges. Bioorg. Med. Chem. Lett 2019, 29, $1555-1564$.

(49) Hughes, J. D.; Blagg, J.; Price, D. A.; Bailey, S.; Decrescenzo, G. A.; Devraj, R. V.; Ellsworth, E.; Fobian, Y. M.; Gibbs, M. E.; Gilles, R. W.; Greene, N.; Huang, E.; Krieger-Burke, T.; Loesel, J.; Wager, T.; Whiteley, L.; Zhang, Y. Physicochemical drug properties associated with in vivo toxicological outcomes. Bioorg. Med. Chem. Lett 2008, 18, $4872-4875$.

(50) Sander, T. Actelion's property explorer, Actelion's Pharmaceuticals Ltd.: Gewerbestrasse 16, 4123 Allschwil, Switzerland, 2001.

(51) Zhao, Y.; Truhlar, D. G. A new local density functional for maingroup thermochemistry, transition metal bonding, thermochemical kinetics, and noncovalent interactions. J. Chem. Phys. 2006, 125, 194101-194118.

(52) Lee, C.; Yang, W.; Parr, R. G. Development of the Colle-Salvetti correlation-energy formula into a functional of the electron density. Phys. Rev. B 1988, 37, 785-789.

(53) Frisch, M. J.; Trucks, G. W.; Schlegel, H. B.; Scuseria, G. E.; Robb, M. A.; Cheeseman, J. R.; Scalmani, G.; Barone, V.; Petersson, G. A.; Nakatsuji, H.; Li, X.; Caricato, M.; Marenich, A. V.; Bloino, J.; Janesko, B. G.; Gomperts, R.; Mennucci, B.; Hratchian, H. P.; Ortiz, J. V.; Izmaylov, A. F.; Sonnenberg, J. L.; Williams-Young, D.; Ding, F.; Lipparini, F.; Egidi, F.; Goings, J.; Peng, B.; Petrone, A.; Henderson, T.; Ranasinghe, D.; Zakrzewski, V. G.; Gao, J.; Rega, N.; Zheng, G.; Liang, W.; Hada, M.; Ehara, M.; Toyota, K.; Fukuda, R.; Hasegawa, J.; Ishida, M.; Nakajima, T.; Honda, Y.; Kitao, O.; Nakai, H.; Vreven, T.; Throssell, K.; Montgomery, J. A., Jr.; Peralta, J. E.; Ogliaro, F.; Bearpark, M. J.; Heyd, J. J.; Brothers, E. N.; Kudin, K. N.; Staroverov, V. N.; Keith, T. A.; Kobayashi, R.; Normand, J.; Raghavachari, K.; Rendell, A. P.; Burant, J. C.; Iyengar, S. S.; Tomasi, J.; Cossi, M.; Millam, J. M.; Klene, M.; Adamo, C.; Cammi, R.; Ochterski, J. W.; Martin, R. L.; Morokuma, K.; Farkas, O.; Foresman, J. B.; Fox, D. J. Gaussian 16 software suite; Gaussian, Inc.: Wallingford CT, 2016. 
(54) Marenich, A. V.; Cramer, C. J.; Truhlar, D. G. Universal Solvation Model Based on Solute Electron Density and on a Continuum Model of the Solvent Defined by the Bulk Dielectric Constant and Atomic Surface Tensions. J. Phys. Chem. B 2009, 113, 6378-6396.

(55) Dennington, R.; Keith, T. A.; Millam, J. M. GaussView, version 6.1; Semichem Inc.: Shawnee Mission, KS, 2016.

(56) Molinspiration Chemoinformatics software, https://www. molinspiration.com.

(57) Daina, A.; Michielin, O.; Zoete, V. SwissTargetPrediction: Updated Data and New Features for Efficient Prediction of Protein Targets of Small Molecules. Nucleic Acids Res. 2019, 47, W357-W364. (58) Osiris property explorer. www.organicchemistry.org/prog/peo/ (accessed date June 20, 2021). 